\title{
Del campo de batalla a las calles: el derecho a la intimidad en la era de los drones ${ }^{* *}$
}

\section{From the battlefield to the streets: The privacy right in the drone era}

SUMARIO

Introducción. I. Del ejército a los ciudadanos. II. Norteamérica y el Gran Hermano. A. Desarrollo normativo norteamericano. B. Desarrollo jurisprudencial. C. Desarrollo legal local. III. La legislación incompleta; el caso colombiano. A. Desarrollo legal del derecho a la intimidad en Colombia. B. Desarrollo jurisprudencial con fundamento en el artículo 15 de la Constitución de 1991. Conclusión.

\section{RESUMEN}

Si bien la tecnología de los drones (desde ahora Unmaned Aircraft Systems o UAS) no es nueva en el mundo militar y se encuentra ampliamente documentada, en los últimos años se ha dado un inesperado incremento en la venta de este tipo de equipos, ahora destinados a actividades civiles o para recreación $\mathrm{y}$ entretenimiento. Esto ha generado problemas jurídicos sin precedentes, especialmente desde el punto de vista de la privacidad, que han hecho necesario que países como Estados Unidos limiten el uso de UAS. El presente artículo tiene como objetivo hacer un análisis del derecho a la intimidad y la privacidad en el uso de los UAS desde la óptica estadounidense y colombiana, planteando conclusiones y posibles caminos a seguir.

* Abogado de la Universidad del Rosario (Colombia), candidato a magíster en Derecho Informático y Propiedad Intelectual de la Universidad Gottfried Wilhelm Leibniz de Hannover (Alemania) y la Universidad de Oslo (Noruega).Contacto: ramirezlopez2790@gmail.com

** Recibido el: 23 de noviembre de 2014, aprobado el: 12 de octubre de 2015.

Para citar el artículo: S. RAMíREZ LóPEZ, Del campo de batalla a las calles: el derecho a la intimidad en la era de los drones, Derecho del Estado n. ${ }^{\circ} 35$, Universidad Externado de Colombia, julio-diciembre de 2015, pp. 181-199. DOI: http://dx.doi.org/10.18601/01229893. n35.07 
PALABRAS CLAVE

Drone, UAS, derecho a la intimidad, protección a la privacidad, vigilancia.

PALABRAS CLAVE DESCRIPTOR

Derecho informático, América Latina, Colombia, Estados Únidos de América.

\begin{abstract}
While drone technology (from now on Unmanned Aircraft Systems or UAS) is not a novelty in the military world and it has been broadly documented, during the last years there has been an unexpected sales increase in this kind of equipment, now for civil, recreation and entertainment purposes. This has generated unprecedented legal problems, especially from the privacy point of view, which has made countries like United States to limit the use of UAS. This article aims to analyze the intimacy and privacy rights in the use of UAS from the United States and Colombian point of view, stating conclusions and possible paths to follow.
\end{abstract}

\title{
KEY WORDS
}

Drone, UAS, intimacy right, protection of privacy, surveillance.

KEY WORDS PLUS

IT Law, Latin America, Colombia, United States of America.

\section{INTRODUCCIÓN}

Los UAS se definen como aeronaves con capacidad de volar sin tripulación, ya sea de maner autónoma u operados de forma remota ${ }^{1}$. Aunque esta categoría incluye aeromodelos y demás equipos de réplicas a escala, en la actualidad se han creado tecnologías que han permitido incorporar nuevas funciones y formas, dando mayor capacidad de maniobrabilidad y muchas veces incorporando equipos de fotografía y de grabación de video. Este tipo de UAS ha despertado preocupaciones desde varios puntos de vista; desde el punto de vista guber2014, p. 28. 
namental y político existen mayores preocupaciones en relación con los usos bélicos y militares, mientras que desde el punto de vista civil y recreativo las preocupaciones se han enfocado en el derecho y la defensa de la privacidad.

El pasado 15 de febrero, la Federal Aviation Administration (desde ahora FAA) de los Estados Unidos de América, propuso un nuevo marco de regulaciones para operar pequeños UAS con fines civiles no recreativos. Si bien este marco aún se encuentra pendiente de diferentes trámites para entrar a regir plenamente ${ }^{2}$, plantea interrogantes en relación con lo que debe y no debe limitarse a la hora de usar UAS. Esta propuesta y las demás normas existentes no subsanan el vacío en la defensa de la privacidad de los ciudadanos, al dejar al arbitrio de otras instancias, especialmente las jurisprudenciales, la determinación de una posible vulneración de derechos. En este sentido, el sistema jurídico norteamericano, determinado por la ausencia de normas que específicamente protejan la privacidad de los ciudadanos frente al uso de UAS, hace especial hincapié en la aplicación directa de los principios consagrados en la Cuarta Enmienda de la Constitución ${ }^{3}$.

En el caso latinoamericano se han tomado muy pocas medidas para actualizar las regulaciones que hacen frente a problemas inéditos generados por los UAS. Salvo el caso de Brasil, que ha tomado la delantera en esta problemática y a la vez cuenta con el mayor número de UAS en la región ${ }^{4}$, ningún otro país latinoamericano ha afrontado el debate directamente, y la mayoría se han limitado a extender el objetivo de normas antiguas, que aunque guardan una estrecha relación pierden de vista las nuevas situaciones que se plantean hoy en día con esta clase de equipos.

Bajo esta óptica, el presente trabajo tiene como finalidad: 1. Analizar los adelantos que se han hecho sobre el particular en un país avanzado en esta problemática, como es Estados Unidos, a fin de determinar si existe realmente una protección para los ciudadanos en casos de intrusiones o pesquisas privadas o gubernamentales que vulneren una expectativa legítima de privacidad; 2. Analizar si existen tales protecciones en un país en donde los adelantos normativos en materia de UAS son prácticamente nulos, como es el caso de Colombia; y finalmente, 3. Plantear una conclusión, partiendo de los peligros derivados de la carencia normativa, y formular una propuesta sobre el camino que debería seguirse al respecto.

2 Section 333 of the FAA Modernization and Reform Act of 2012.

3 J. O'BRIEN. Warrantless Government Drone Surveillance: A Challenge to the Fourth Amendment. Marshall J. Information Technology \& Privacy Law, vol. Xxx, 2013, p. 168.

4 M. CAWLEY. Drone use in Latin America: Dangers and Opportunities. Insight Crime. 18 de abril de 2014. Recuperado de: http://www.insightcrime.org/news-analysis/drone-use-inlatin-america-dangers-and-opportunities 


\section{DEL EJÉRCITO A LOS CIUDADANOS}

Durante los últimos 5 años, el mercado de UAS ha crecido exponencialmente tanto en ámbitos militares como civiles. La FAA, conservadoramente, estima que los UAS privados constituirán una industria de 90 billones de dólares durante la próxima década ${ }^{5}$, y se cree que entre los años 2015 y 2025 la integración de UAS a la economía norteamericana en aspectos como la agricultura y la seguridad pública será de 82.1 billones, creando alrededor de 103.776 empleos $^{6}$.

A pesar del reciente interés despertado por estos equipos, los UAS no son un fenómeno nuevo. Su desarrollo se remonta a la Primera Guerra Mundial, en donde el ejército de Estados Unidos usó modelos rústicos y poco fiables para realizar operaciones peligrosas en territorio enemigo ${ }^{7}$. En el transcurso y luego de la Segunda Guerra Mundial, el ejército estadounidense hizo uso de la tecnología de control remoto para realizar operaciones en territorios como Vietnam y la Unión Soviética ${ }^{8}$. Sin embargo, es solo con la operación Tormenta del Desierto y el conflicto en los Balcanes que los UAS empiezan a ganar interés y a ser reconocidos por el público en general ${ }^{9}$.

Hoy en día es posible encontrar un sinnúmero de modelos de UAS, de diferentes tamaños y presentaciones, dependiendo de las necesidades. Los UAS pueden encontrarse como globos, dirigibles, planeadores, cometas, planeadores con alerón, propulsados con motores de jet, con dos o cuatro rotores, helicópteros, multicópteros, entre otros ${ }^{10}$. En este sentido, la denominación UAS es amplia y no se limita a una forma o tamaño sino que comprende cualquier tipo de aeronave no tripulada que puede ser operada remotamente o puede funcionar de manera autónoma.

5 L. DownEs. America can't lead the world in innovation if the FAA keeps dragging its feet on drone rules. The Washington Post, 8 de octubre de 2014. Recuperado de: http://www.washingtonpost.com/blogs/innovations/wp/2014/10/08/america-cant-lead-the-world-in-innovationif-the-faa-keeps-dragging-its-feet-on-drone-rules/

6 D. Jenkins y B. VAsigh. The Economic Impact of Unmanned Aircraft Systems Integration in the United States. Association of Unmanned Vehicle Systems International (AUVSI). Marzo de 2014. Recuperado de https://fortunedotcom.files.wordpress.com/2013/05/new_economic_report_2013_full.pdf

7 K. P. Valanis. Advances in Unmanned Aerial Vehicles: State of the Art and the Road to Autonomy. Dordrecht: Springer Science \& Business Media, 2007, p. 3.

8 C. Schlag. New privacy battle: How the expanding use of drones continues to erode our concept of privacy and privacy rights. Pittsburgh Journal of Technology Law \& Policy. Vol. 13, 2013, p. 4 .

9 VALANIS, ob. cit.

10 A. GRUEN. From toys to tools: Unmanned aerial vehicles. GeoInformatics Magazine. Vol. 15, enero-febrero de 2012, p. 14. 
La creciente masificación de los UAV y las características que les permiten realizar labores peligrosas para las persona han traído un sinnúmero de consecuencias y problemas jurídicos totalmente nuevos.

Desde un punto de vista militar y político podemos apreciar que, desde años atrás, los países occidentales han disminuido drásticamente el desarrollo de aviones de combate y el entrenamiento de pilotos para reemplazarlos por operadores de UAS ${ }^{11}$. Esta ausencia casi total de personal militar en las áreas de combate ha traído como inesperada consecuencia que se diluyan los límites para realizar operaciones bélicas, pues eliminando la incomodidad política que traen las bajas humanas en áreas de combate, el gobierno estadounidense ha tomado una postura más laxa a la hora de realizar bombardeos en territorio extranjero sin necesidad de la aprobación del Congreso ${ }^{12}$.

Ha sido tan extendido el uso de de estos equipos en operaciones del ejército norteamericano que hasta el año 2015 se han realizado alrededor de 413 operaciones de guerra por medio de UAS en Pakistán, posiblemente más de 200 en Yemen y cerca de 20 en Somalia ${ }^{13}$, generando centenares de muertos, de los cuales la mayor parte han pasado desapercibos para los medios por la ausencia de bajas norteamericanas. Sin embargo, este no es un fenómeno que se limite a Estados Unidos. Ya para el año 2013, 15 países de América Latina poseían o estaban por adquirir oficialmente UAS ${ }^{14}$. Es de esperarse que esa cifra haya aumentado exponencialmente hasta el día de hoy.

En el escenario regional se han dado importantes desarrollos, especialmente en Brasil, en donde alianzas con Israel han dado lugar a un incremento sustancial en el uso de UAS. La Copa Mundial de Futbol de 2014 fue un interesante campo de prueba para el uso de UAS por parte de los medios de comunicación, logrando despertar ciertas preocupaciones en materia de privacidad ${ }^{15}$.

11 P. W. Singer. Do drones undermine Democracy? The New York Times. 21 de enero de 2012. Recuperado de: http://www.nytimes.com/2012/01/22/opinion/sunday/do-drones-underminedemocracy.html?pagewanted=all\&_r $=0$

12 SINGER, ob. cit.

13 The Bureau Investigative Journalism. Get the Data: Drone Wars: Casualty Estimates (actualizado a 10 de febrero de 2015). Recuperado de: http://www.thebureauinvestigates.com/ category/projects/drones/drones-graphs/ (Consultado por última vez el 13 de marzo de 2015).

14 Comisión Interamericana de Derechos Humanos. Anexo al Comunicado de Prensa CIDH: Culmina el 149 periodo de sesiones, 8 de noviembre de 2013. Recuperado de: http://www.oas. org/es/cidh/prensa/comunicados/2013/083A asp

15 Durante la Copa del Mundo Brasil 2014, el entrenador francés Didier Deschamps denunció intromisiones de drones en entrenamientos del equipo. Véase Otros autores. La polémica por los drones espías llegó al mundial. Telam, 17 de junio de 2014. Recuperado de: http://www. telam.com.ar/notas/201406/67628-la-polemica-por-los-drones-espias-llego-al-mundial.html 
Colombia ha avanzado poco en materia de UAS, a pesar de que el Gobierno ya hace uso de esta clase de equipos. Desde el año 2014, UAS israelitas custodian las fronteras en busca de actividades subversivas, mientras que los modelos Navigator X2, de tipo civil, e IRIS UAS, de carácter militar, han sido desarrollados y fabricados localmente ${ }^{16}$.

Pese a la cobertura que desde varios puntos de vista se ha dado a los UAS, aún no existe total claridad sobre el potencial, los peligros y los caminos a seguir en este nuevo escenario global, en donde muchos gobiernos están reemplazando a sus pilotos por operadores de UAS y las personas del común tienen un acceso nunca antes visto a esta clase de equipos. Las ventajas, facilidades tecnológicas y el hecho de que estas tecnologías sean cada vez más asequibles han generado un aumento inusitado en la vigilancia a los ciudadanos por parte de gobiernos y civiles, pero a su vez han generado un rechazo sistemático de las sociedad hacia cualquier intrusión gubernamental o privada que pueda vulnerar lo que se considera privado e íntimo.

A partir de este escenario contradictorio que nos plantea el mundo contemporáneo, vale la pena analizar las regulaciones que se han proferido sobre el particular. En este aspecto, Estados Unidos ofrece un interesante punto de partida para el análisis académico y puede dar luces sobre el futuro que debe seguir la normativa colombiana y latinoamericana.

\section{NORTEAMÉRICA Y EL GRAN HERMANO}

Con el espacio aéreo más ocupado y complejo del mundo, es comprensible que Estados Unidos sea uno de los mayores reguladores en materia de UAS. A esto hay que adicionarle el hecho de que Estados Unidos ha sido el país que mayor uso militar le ha dado a los UAS, con casi un siglo de historia. En la actualidad sigue siendo el territorio en donde más se usan los UAS, con un aumento exponencial en materia de operaciones militares y de seguridad interior, especialmente en la vigilancia de fronteras y la lucha contra los carteles mexicanos ${ }^{17}$. Sin embargo, vale la pena analizar si las medidas tomadas en Estados Unidos son suficientes para realizar una protección efectiva de la privacidad de los ciudadanos.

\section{A. Desarrollo normativo norteamericano}

En materia normativa, la FAA ha dividido las disposiciones concernientes a UAS de acuerdo al tipo de uso que se le dé al equipo. En primer lugar, encon-

16 J. Quintero. Conozca los drones Made in Colombia. El Tiempo. 21 de abril de 2013. Recuperado de: http://www.eltiempo.com/archivo/documento/CMS-12753146

17 O'BRIEN, ob. cit., p. 163. 
tramos las Operaciones Públicas (Public Operations) realizadas directamente por el gobierno. Se ha entendido que los UAS son Aeronaves Públicas (Public Aircrafts) siempre y cuando sean propiedad del gobierno o las fuerzas armadas, operadas por estas o por cualquier persona para fines no comerciales ${ }^{18}$.

No se hace ninguna distinción entre aeronaves operadas remotamente y aquellas con pasajeros. El desconocimiento, no necesariamente involuntario, de las características de UAS en operaciones públicas implica que no existen reglamentaciones específicas en relación con la privacidad y el manejo de información, pues no se tienen en cuenta las distinciones y particularidades de los UAS que las hacen especialmente aptas para la vigilancia y el espionaje. Una posición ampliamente aceptada señala que la FAA no se encuentra facultada o preparada para dictar normas en relación con la privacidad individual ${ }^{19}$.

Por otra parte, la FAA contempla las Operaciones Civiles, entendiendo para estos fines como aeronaves civiles a todas aquellas que no cumplan con los criterios para ser catalogadas como públicas ${ }^{20}$. Como punto importante a resaltar, se exige una autorización SAC (Special Airworthiness Certificate) otorgada por la FAA, salvo que la Secretaría de Transporte determine, caso por caso, que una operación comercial a través de UAS no representa un riesgo para los usuarios, el sistema nacional aeroespacial, el público y/o no amenaza la seguridad nacional ${ }^{21}$. Sin embargo, estas disposiciones serán posiblemente modificadas a través del marco de regulaciones propuesto el pasado 15 de febrero de 2015 por la FAA, en donde se incluye la necesidad de mantener contacto visual con la aeronave y el hecho de denominar al usuario como operador que requiere un certificado, pero flexibilizando y no exigiendo la autorización SAC.

Como último punto, se contempla el uso recreativo, el cual se encuadra dentro de las normas concernientes al aeromodelismo ${ }^{22}$. Resalta el hecho de que las normas prohíben a la FAA promulgar cualquier tipo de regulación en relación con los aeromodelos, siempre y cuando se trate de aeronaves que se utilicen para fines recreativos, no afecten la seguridad, no pesen más de 55 libras y no interfieran con el vuelo de aeronaves tripuladas o sean voladas cerca de un aeropuerto. Esta disposición elimina la capacidad normativa de la FAA para UAS de entretenimiento.

18 United States Code (49 U.S.C.) $\S \$ 40102$ (a)(41).

19 O'BRIEN, ob. cit., p. 167.

20 United States Code (49 U.S.C.) $§ \S 40102$ (a)(16).

21 Section 333 of the FAA Modernization and Reform Act of 2012 (FMRA).

22 Section 336 - Public Law 112-95. 
Ahora bien, podemos apreciar que las normas federales no han incluido y no planean incluir normas en relación con el derecho a la privacidad de los individuos, consagrado este último en la Cuarta Enmienda de la Constitución de Estados Unidos ${ }^{23}$. No obstante, la jurisprudencia ha sentado su posición a través de diferentes decisiones que se han encargado de definir los parámetros de la defensa a la privacidad, cuando está siendo vulnerada a través del uso de instrumentos tecnológicos de vigilancia. Lo anterior con fundamento en la defensa de la Cuarta Enmienda contra pesquisas arbitrarias o injustificadas por parte del gobierno o de privados.

\section{B. Desarrollo jurisprudencial}

En primer lugar, vale la pena señalar que la Constitución de Estados Unidos incluye una protección frente a intrusiones arbitrarias del gobierno, pero no existen disposiciones legales que incluyan esta misma protección cuando se trata de intrusiones realizadas por particulares. Sin embargo, la jurisprudencia ha equiparado conceptos utilizados para los casos gubernamentales, aplicando conceptos como la expectativa razonable de privacidad, para proteger a las personas de pesquisas arbitrarias realizadas por entes privados o personas particulares $^{24}$.

La doctrina norteamericana concuerda en la existencia de dos momentos diferentes en cuanto a tendencia jurisprudencial se refiere. La primera y más antigua tiene su origen en la vigilancia a través de la intervención telefónica (wiretapping) y las escuchas secretas (eavesdropping) ${ }^{25}$.

En el caso Goldman v. United States de $1942^{[26]}$, oficiales de la ley utilizaron aparatos tecnológicos para escuchar conversaciones telefónicas que el indiciado había realizado en un cuarto contiguo sin una orden o autorización judicial. En este caso y en otros como Olmstead v. United States ${ }^{27}$, la Corte encontró que no se había presentado una violación a la Cuarta Enmienda, en tanto no se había realizado una intrusión física en el lugar en donde se había

23 La cuarta enmienda protege a los habitantes de Estados Unidos de pesquisas y aprehensiones, cuando estas son arbitrarias. Textualmente: "El derecho de los habitantes de que sus personas, domicilios, papeles y efectos se hallen a salvo de pesquisas y aprehensiones arbitrarias, será inviolable, y no se expedirán al efecto mandamientos que no se apoyen en un motivo verosímil, estén corroborados mediante juramento o protesta y describan con particularidad el lugar que deba ser registrado y las personas o cosas que han de ser detenidas o embargadas".

24 D. L. Braumer y J. C. PoindeXTer. Cyberlaw and E-Commerce. New York, McGrawHill Irwin, 2002, pp. 157-158.

25 O'BRIEN, ob. cit., p.171.

26 Goldman v. United States, US Supreme Court (27 de abril de 1942).

27 Olmstead v. United States, us Supreme Court (4 de junio de 1928). 
llevado a cabo la conversación telefónica. Esta tendencia limitaba la privacidad del investigado a la presencia física e intrusiva de los instrumentos de vigilancia en la locación del investigado.

Dicha tendencia jurisprudencial sería modificada en 1967, a través del caso Katz v. United States ${ }^{28}$. En dicho caso, el FBI recopiló conversaciones telefónicas adhiriendo instrumentos de escucha secreta a una cabina telefónica en donde el indiciado realizaba actividades ilegales. A pesar de que la Corte Suprema encontró que las escuchas habían sido ilegales, modificó el criterio de violación a la Cuarta Enmienda de intrusión física por el de expectativa razonable de privacidad.

Bajo la doctrina traída por el caso Katz v. United States, la expectativa de privacidad del vigilado debe ser razonable, lo cual se logra satisfaciendo dos condiciones; la persona debe exhibir una expectativa subjetiva de privacidad demostrando su interés de mantener un espacio en la intimidad; y la expectativa debe ser aceptada como razonable por la sociedad ${ }^{29}$.

Esta tendencia ha sido reiterada a través de otros casos. En el caso Kyllo v. United States $^{30}$, agentes del Departamento de Interior utilizaron cámaras térmicas para detectar lámparas destinadas al cultivo de marihuana en el hogar de Danny Kyllo.

Respecto de la viabilidad del uso de este tipo de tecnologías, la Corte señaló que se había llevado a cabo una búsqueda que vulneraba el derecho a la privacidad del indiciado en cuanto existía una expectativa legítima de privacidad, al haberse utilizado instrumentos que exploraban detalles de la casa que hubieran sido desconocidos sin una intervención física, para lo cual se requería una orden judicial.

En el caso Dow Chemicals v. United States ${ }^{31}$, el peticionario, dueño de una planta de dos mil acres, se negó a la solicitud de visita realizada por la Agencia de Protección del Ambiente (Environmental Protection Agency, desde ahora EPA). Por este motivo, EPA utilizó equipos de fotografía aérea para estudiar la planta. La Corte determinó que no se había violado la Cuarta Enmienda, dado que las plantas inspeccionadas eran visibles a simple vista, lo que implicaba que no era posible tener una expectativa legítima de privacidad sobre las mismas.

Este caso también incorpora una doctrina importante para la jurisprudencia norteamericana, llamada la doctrina de campo abierto (The open field

28 Katz v. United States, us Supreme Court (18 de diciembre de 1967).

29 J. Villasenor. Observation from above: Unmanned Aircraft Systems and Privacy. Harvard Journal of Law \& Public Policy, Vol. 36, 2013, p. 36.

30 Kyllo v. United States, Us Supreme Court (11 de junio de 2001).

31 Dow Chemicals v. United States, US Supreme Court (19 de mayo de 1986). 
doctrine), inicialmente instaurada por el caso Hester v. United States ${ }^{32}$ y posteriormente desarrollada por otros casos, la cual plantea la dificultad de alegar una expectativa de privacidad sobre situaciones que ocurren en espacios abiertos y visibles al público.

Finalmente, el caso Smith v. Maryland ${ }^{33}$ también es ilustrativo. En este caso, la policía instaló en el teléfono del peticionario un instrumento que permite saber los números marcados (Pen register). Frente al argumento de que dicho instrumento vulneraba los derechos consagrados en la Cuarta Enmienda, la Corte Suprema determinó que cualquier persona al usar el teléfono exponía el número marcado a la compañía telefónica, concluyendo que la sociedad no se encuentra preparada para reconocer una expectativa legítima de privacidad sobre un número telefónico, no satisfaciendo el segundo criterio de la expectativa de privacidad ${ }^{34}$.

En suma, junto con otras decisiones jurisprudenciales relacionadas, se ha dado lugar a una postura en donde se entiende vulnerada la Cuarta Enmienda con ocasión de pesquisas arbitrarias cuando existe una expectativa razonable de privacidad respecto del objeto que está siendo indagado. Sin bien se le da importancia a la intervención física del investigado, dicha intervención tiene fundamento en el hecho de que exista una expectativa de privacidad.

\section{Desarrollo legal local}

Como parte de la autonomía a los Estados, existen normas específicas que consagran conductas delictuales relacionadas con el vouyerismo. Algunas, como una del estado de Arkansas, consagran el delito de video vouyerismo, prohibiendo las filmaciones o las fotografías tomadas a una persona, siempre que no exista consentimiento y haya una expectativa de privacidad ${ }^{35}$. El estado de Maine consagra normas similares, sancionando conductas que consistan en entrar en una propiedad o instalar equipos para escuchar u observar a una persona dentro de su espacio privado ${ }^{36}$.

Sin embargo, no todas las legislaciones son amplias, muchas se limitan a criminalizar actuaciones de este tipo siempre y cuando exista una finalidad sexual. Por ejemplo, la legislación del estado de Colorado criminaliza la in-

32 S.A. SALmon. Search and Seizure: Open field doctrine in lights of Katz - United States v. Basile. Arizona State Law Journal, 2-3, 1978, p. 366.

33 Smith v. Maryland, US Supreme Court (20 de junio de 1979).

34 O'BRIEN, ob. cit., p. 188.

35 Ark. Code Ann. § 5-16-101 (2008).

36 Me.Rev. Stat. Ann. tit. 17-A, § 511 (2008). 
vasión a la privacidad cuando se toman fotografías de las partes íntimas de otra persona sin su autorización y cuando exista una expectativa legítima de privacidad $^{37}$. Provisiones similares están incluidas en las legislaciones de los estados de Hawai ${ }^{38}$, Idaho ${ }^{39}$ y Washington ${ }^{40}$, entre otros.

Lo que se evidencia es que no existen normas federales en Estados Unidos que prohíban o limiten la vigilancia gubernamental, y por extensión civil, sin una autorización. Más aún, se ha dado a los estados la autonomía para reglamentar el espionaje y la vigilancia no autorizada, pero estas legislaciones no se encuentran unificadas, lo que genera un concepto fragmentado e inseguro jurídicamente, muchas veces limitado a criminalizar conductas con una connotación netamente sexual, desconociendo otras realidades.

La fragmentación, por una parte, y la falta de regulación federal, por otra, han generado una aplicación directa, aunque en muchos casos ambigua de la Cuarta Enmienda por parte de las Cortes norteamericanas ${ }^{41}$. Si bien Estados Unidos cuenta con conceptos pertinentes para la correcta protección del derecho a la privacidad de los ciudadanos frente a los UAS, y aunque la Corte Suprema ha creado precedentes importantes, existe una falta de unificación normativa, que no permite hablar de seguridad jurídica en un plano federal.

\section{LA LEGISLACIÓN INCOMPLETA; EL CASO COLOMBIANO}

En Colombia, el uso de UAS, aunque no encuentra una regulación específica, sí está contemplado por vía analógica en normas preexistentes. Para estos fines, se debe acudir al Reglamento Aeronáutico Colombiano (en adelante RAC), el cual incluye un capítulo dedicado al aeromodelismo, en donde se señala que este tipo de equipos no serán considerados como aeronaves, no estando sometidos a las disposiciones aeronáuticas generales, aunque limitados bajo ciertos parámetros ${ }^{42}$.

La norma también hace referencia al uso de aeromodelos para fines deportivos o recreativos y para fines civiles. Se exige que cualquier actividad deportiva a través de aeromodelos o equipos similares se haga mediando una afiliación a un club especializado, y que dicho club esté inscrito en Coldeportes ${ }^{43}$. Sin

38 Haw. Rev. Stat. Ann. \$ 711-1110.9 (2008).

39 Idaho Code Ann. § 18-6609 (2008).

40 Wash. Rev. Code Ann. § 9A.44.115 (2008).

41 O'BRIEN, ob. cit., p. 168.

42 Numeral 4.25.8. del RAC 4.

43 Numeral 4.25.8.1. del RAC 4. 
embargo, también se contemplan y se asimilan al aeromodelismo las operaciones de equipos de vuelo no tripulados y radiocontrolados, cuando su operación no tenga fines deportivos, como es en el caso de la fotografía, la teledetección o la televisión ${ }^{44}$.

El RAC incluye limitantes al uso de aeromodelos, enfocados primordialmente en evitar operaciones irresponsables que puedan acarrear un daño a la propiedad privada o a la integridad de las personas ${ }^{45}$. Aunque no debe menospreciarse la importancia del uso responsable de UAS para evitar daños, es claro que se trata de normas que pierden el punto de vista en relación con el potencial, tanto positivo como negativo de los UAS. La exigencia de afiliación de los equipos a clubes deportivos, cuando se tiene una finalidad deportiva o recreativa, aunque lógica para aeromodelos, no contempla la realidad actual de los UAS, cada vez más económicos y populares, lo que la hace una disposición probablemente inefectiva que desconoce la realidad.

\section{A. Desarrollo legal del derecho a la intimidad en Colombia}

Colombia también ha dado pasos importantes en el desarrollo del derecho a la intimidad con fundamento en el artículo 15 de la Constitución Política de 1991, que garantiza el derecho a la intimidad personal y familiar frente a actuaciones estatales y privadas. Este derecho es específicamente garantizado desde diferentes ópticas, tales como el tratamiento, recolección y circulación de datos o la privacidad de la correspondencia y la comunicación privada.

Vale la pena señalar que mediante el Acto Legislativo 02 de 2003, declarado inexequible por la Corte Constitucional en la sentencia C-816 de 2004, se intentó conminar al Congreso de la República a expedir una ley estatutaria que regulara casos en donde se permitieran las interceptaciones y registros de correspondencia o comunicaciones privadas sin orden judicial, con fundamento en la prevención de actos terroristas. La decisión jurisprudencial que declara inexequible el mencionado acto legislativo fue consagrada en la Ley Estatutaria 1621 de 2013, en donde se señala que toda interceptación de comunicaciones se sujetará a lo dicho en el artículo 15 de la Constitución Política y en el Código de Procedimiento Penal, y que solo será efectiva dentro de un proceso judicial ${ }^{46}$.

Lo anterior consagra legal y constitucionalmente a Colombia como un Estado garantista en materia de privacidad, lo cual es acertado en cuanto

44 Numeral 4.25.8.2. del RAC 4.

45 Numeral 4.25.8. del RAC 4.

46 Ley Estatutaria 1621 de 2013, arts. 17 y 44. 
limita facultades que en otro caso podrían prestarse para arbitrariedades. Sin embargo, el lenguaje de estas normas parece limitar su alcance a las interceptaciones de comunicaciones, como respuesta a diferentes escándalos recientemente ocurridos en el país ${ }^{47}$.

El Código Penal (Ley 599 de 2000), en sus capítulos VI y VII, contiene delitos relacionados con la inviolabilidad de habitación o sitio de trabajo y la interceptación de comunicaciones. Los artículos 189 a 191 consagran delitos consistentes en la introducción arbitraria o abusiva en la habitación o sitio de trabajo para grabar, fotografiar o filmar. Los artículos 192 a 197 consagran la interceptación de comunicaciones a través de diferentes medios.

Aun así, es posible formular objeciones respecto a los artículos 189 a 191, en tanto limitan su aplicación a una intrusión en el sitio de habitación o de trabajo, dejando atrás la vigilancia realizada por medios que no impliquen irrumpir físicamente en un espacio. Los UAS son un equipo efectivo para realizar vigilancias ilegítimas sin la necesidad de una intrusión física en una propiedad privada.

\section{B. Desarrollo jurisprudencial con fundamento en el artículo 15 de la Constitución de 1991}

Tal y como ocurre con la Cuarta Enmienda en Estados Unidos, la Corte Constitucional de Colombia ha formulado diferentes tesis para la protección del derecho a la intimidad con base en el artículo 15 de la Constitución Política, algunas de las cuales son pertinentes para el presente análisis.

Desde el año 1992, la sentencia T-414 reconoció el derecho a la intimidad como un derecho erga omnes que legitima a cualquier persona a ejercerlo por el hecho de ser persona. De igual manera, se reconoció la prevalencia del derecho a la intimidad por sobre el derecho a la información, en cuanto para la Corte la intimidad tiene una clara conexión con la dignidad humana, por lo que solo puede ser limitada cuando el interés general lo dicte.

En la sentencia T-696 de 1996 se examina el caso de un noticiero, que a través de cámaras interceptó una nota privada escrita por el accionante durante una sesión plenaria de la Cámara de Representantes. En primer lugar, este caso es un referente para la Corte Constitucional al señalar que el derecho

47 Los actos referidos al primero de los casos, dado a conocer a principios del año 2009 por la revista Semana, por el cual se emitió condena contra la ex Directora del DAS, María del Pilar Hurtado, y que tiene en la mira pública al ex Presidente Álvaro Uribe, se realizaron desde las oficinas del DAS, en donde se interceptaron comunicaciones de algunos de los líderes de la oposición al gobierno. Véase Otros autores. El DAS sigue grabando. Semana, 21 de febrero de 2009. Recuperado de: http://www.semana.com/nacion/articulo/el-das-sigue-grabando/100370-3 
a la intimidad puede ser vulnerado de tres maneras: "La primera de ellas es la intrusión o intromisión irracional en la órbita que cada persona se ha reservado; la segunda, consiste en la divulgación de los hechos privados; y la tercera, finalmente, en la presentación tergiversada o mentirosa de circunstancias personales, aspectos los dos últimos que rayan con los derechos a la honra y al buen nombre".

La Corte Constitucional consagra la inviolabilidad de la correspondencia, la cual es privada en cuanto la voluntad del remitente así se exprese, independientemente de que el sitio de correspondencia sea público o privado. Así mismo, el derecho a la información de un noticiero implica también el respeto al ejercicio de los derechos fundamentales de todos los ciudadanos, por lo que debe a su vez coexistir con el derecho a la intimidad de quien sea sujeto de una noticia.

En la sentencia T-517 de 1998, la Corte Constitucional examinó el caso del interno de una penitenciaría, que a través de acción de tutela alegaba que los guardias violaban su derecho a la intimidad, en cuanto escuchaban las conversaciones telefónicas que tenía con su esposa. En este caso, la Corte acudió al concepto de la esfera espacial, haciendo necesaria una diferenciación según el grado de privacidad que tiene cada espacio, en un análisis casuístico, tema que fue más ampliamente abordado por la sentencia T-407 de 2012.

En la mencionada sentencia T-407, la Corte analizó la viabilidad de instalar cámaras de seguridad en las aulas de clase de una institución educativa. En dicha decisión, la Corte consagró la posibilidad que tiene el Estado de limitar la privacidad cuando las razones obedezcan al interés general. Nuevamente, la Corte acudió a las esferas de privacidad de un espacio, para sustentar su tesis, e hizo una diferenciación entre espacio público, espacio privado y espacio semi-público y semi-privado.

El espacio público es el destinado a "la circulación, la recreación, la instalación de servicios públicos, de preservación de obras públicas, y en general todas las zonas en las que prevalezca el interés y las necesidades colectivas, sobre las particulares, en relación con su uso y disfrute"48.

Por el contrario, en el espacio privado, las personas desarrollan libremente su personalidad e intimidad. Se trata de un espacio que requiere respeto casi absoluto, en tanto las actuaciones no salgan de la esfera privada o afecten a terceros. Sin embargo, para la Corte Constitucional existen ciertos grados de privacidad, de acuerdo al tipo de espacio cerrado del que se trate, pues no puede predicarse la misma protección sobre el domicilio de una persona que sobre su lugar de trabajo, en donde se concretan relaciones públicas, recono- 
ciéndose diferentes esferas de privacidad. Se reconoce igualmente el hecho de que la intimidad y los espacios privados son una convención social que cambia con el tiempo.

Así pues, la Corte habla de los espacios semi-públicos y semi-privados, como resultado de la idea de que existen diferentes esferas de privacidad que no pueden ser incluidas en solo dos categorías.

La Corte parte de la idea de que el individuo cede parte de su interioridad hacia el conocimiento público y que, de acuerdo al grado en que se realice este acto, se puede determinar el nivel de intimidad que recae sobre un espacio $^{49}$. Espacios intermedios en el nivel de intimidad que tienen elementos tanto públicos como privados son los centros educativos, restaurantes, oficinas, almacenes, etc. Sin embargo, estos lugares que corresponden a niveles intermedios de intimidad no son equiparables y el nivel de protección a la intimidad dependerá en cada caso. Se trata de una doctrina complicada y casuística, que exige un análisis judicial en casi cualquier caso, especialmente si la protección se pide sobre un espacio semi-privado o semi-público.

Decisiones más recientes de la Corte Constitucional han reconocido la inviolabilidad del derecho a la intimidad y la correspondencia, pero limitada a motivos de interés general y al cumplimiento de requisitos legales. La sentencia C-881 de 2014, al analizar la exequibilidad del artículo 54 de la Ley 1453 de 2011, el cual otorga facultades a los fiscales para realizar seguimientos pasivos a indiciados o imputados con el fin de conseguir información útil para la investigación, determinó que el derecho a la intimidad puede ser objeto de limitaciones, si existen razones de interés general, legítimas y debidamente justificadas.

Dicha sentencia igualmente señala que la existencia de un espacio exento de cualquier intromisión o intervención arbitraria hace parte del contenido básico del derecho fundamental a la intimidad, y reitera la distinción entre espacio privado, público y semi-privado y semi-público. En todo caso, la sentencia es clara en señalar que el simple hecho de ingresar en el espacio privado de una persona constituye una vulneración a su derecho a la intimidad, sin que sea necesario demostrar la recolección de información privada o la divulgación de dato alguno.

Finalmente, vale la pena mencionar la sentencia T-277 de 2015, que analizando la posibilidad de ordenar la rectificación y eliminación de información periodística de una casa editorial y de motores de búsqueda en línea, señaló la estrecha relación del derecho a la honra y al buen nombre con el derecho a la intimidad, en la medida en que la limitación a la injerencia de terceros en 
esferas privadas busca proteger el buen crédito de los individuos. Se resalta la importancia de la divulgación de datos verídicos no oprobiosos, puesto que el descrédito de la imagen pública de una persona tiene vital importancia para su vida social y laboral.

\section{CONCLUSIÓN}

Vistas las posturas actualmente asumidas en Estados Unidos y en Colombia, se evidencia que no existe hasta el momento una regulación que proteja el derecho a la privacidad en el uso de UAS. Si bien se han realizado esfuerzos encaminados a regular muchos de los aspectos relacionados con la seguridad, la protección de la propiedad y la integridad de las personas frente al uso irresponsable de UAS, otros temas relacionados con derechos inherentes se han dejado atrás.

En Colombia encontramos que el derecho a la privacidad y a la intimidad no ha sido un tema que haya encontrado eco en el Congreso de la República, salvo en casos muy específicos, relacionados con la inteligencia y la contrainteligencia estatal, como en el caso de la Ley Estatutaria 1621 de 2013.

En otros casos legales colombianos, como es el de los delitos contra la inviolabilidad de habitación o sitio de trabajo del Código Penal, se han incluido medidas insuficientes para proteger a las personas de violaciones indebidas a su derecho a la intimidad. Al igual que la doctrina de la Corte Constitucional, estos delitos implican una intrusión física en un espacio determinado, como es el sitio de habitación o el lugar de trabajo. Tales disposiciones pueden resultar inservibles en muchos casos, especialmente si se trata de UAS, los cuales tienen la posibilidad de realizar actividades ilegítimas de vigilancia sin invadir físicamente un espacio.

Por lo demás, tanto en Estados Unidos como en Colombia el derecho a la privacidad y a la intimidad tiene consagración constitucional y protección generalmente jurisprudencial. Sin embargo, vale la pena preguntarse si esta protección netamente jurisprudencial, casuística y cambiante derivada de normas amplias como la Cuarta Enmienda estadounidense y el artículo 15 de la Constitucional Política de Colombia es suficientemente efectiva e inmediata para el uso actual de UAS.

La doctrina norteamericana ha reconocido la importancia de diferenciar el tipo de aeronave de acuerdo con sus habilidades técnicas y tecnológicas ${ }^{50}$. Las normas sobre UAS generalmente han extendido disposiciones relacionadas con el aeromodelismo, lo que implica ignorar algunas de las características que 
corresponden específicamente a UAS modernos. Por ejemplo, los modelos clásicos de aeromodelismo, en general representaciones de aeroplanos a escala, no gozan de la maniobrabilidad de un UAS moderno y de hélices, que le permite acceder a sitios difíciles y mantenerse estático por un tiempo prolongado. En este escenario, una delimitación precisa de las características actuales de aeronaves tipo UAS permite dar claridad sobre las posibilidades que ofrecen este tipo de equipos, a su vez brindando claridad respecto de las posibles formas en que podrían violarse los derechos a la privacidad de los ciudadanos.

En el plano jurisprudencial, la Corte Constitucional ha hecho especial énfasis en el espacio y su carácter de público o privado, para determinar la violación del derecho a la intimidad. Sin embargo, debemos preguntarnos de qué manera estas delimitaciones del espacio son suficientes para proteger la intimidad de una persona vigilada. Una persona puede ser captada en imágenes por medio de UAS a través de su ventana sin invadir su espacio privado de manera física. Pero cuando esta práctica es constante, continua y se convierte en vigilancia, la misma ventana puede revelar detalles íntimos de la vida personal o familiar del vigilado, sin invadir su espacio privado. Casos como estos hacen pensar que, además de la necesidad de delimitar espacios por su carácter público y privado, debe adicionarse, de forma complementaria, una expectativa legítima de privacidad, como la planteada por la jurisprudencia norteamericana desde el caso Katz v. United States. De esta manera resulta acertado considerar de manera conexa, no solo las características privadas o públicas de un espacio, sino la expectativa que tiene una persona de estar determinando su vida a su antojo, cuando con su actuar no afecte a terceros.

En el tema normativo colombiano, podemos encontrar las mismas falencias endilgadas a decisiones jurisprudenciales. En efecto, el uso de UAS puede dejar sin base las normas que busquen proteger el derecho a la intimidad de una persona, si esta protección se basa exclusivamente en la intrusión física en un espacio determinado, por lo que las normas penales pueden resultar inservibles en ciertos casos. Más aún, esta falencia puede ser una consecuencia del hecho de que no existe una clara delimitación de los equipos UAS.

En resumen, una delimitación clara de las características de las aeronaves, junto con un cambio en el concepto de intimidad que incluya la expectativa legítima de privacidad, debe redundar en la creación de nuevas normas, en donde el legislador contemple de forma amplia e informada las posibilidades ofrecidas por los UAS.

BIBLIOGRAFÍA

Agruen, A. From Toys to Tools: Unmanned Aerial Vehicles. GeoInformatics Magazine. Vol. 15, enero-febrero de 2012, p. 14. 
ARK. CODE ANN. § 5-16-101 (2008).

Braumer, D. L. y J. C. PoindeXter. Cyberlaw and E-Commerce. New York, McGraw-Hill Irwin, 2002.

CAWley, M. Drone use in Latin America: Dangers and Opportunities. Insight Crime, 18 de abril de 2014. Recuperado de: http://www.insightcrime.org/news-analysis/drone-use-inlatin-america-dangers-and-opportunities

Colo. Rev. Stat. § 18-3-404 (2008).

Comisión Interamericana de Derechos Humanos. Anexo al Comunicado de Prensa CIDH: Culmina el 149 periodo de sesiones, 8 de noviembre de 2013. Recuperado de: http://www. oas.org/es/cidh/prensa/comunicados/2013/083A.asp

Corte Constitucional de Colombia, Sentencia C-816 de 2004.

Corte Constitucional de Colombia, Sentencia C-881 de 2014.

Corte Constitucional de Colombia, Sentencia T-277 de 2015.

Corte Constitucional de Colombia, Sentencia T-407 de 2012.

Corte Constitucional de Colombia, Sentencia T-414 de 1992.

Corte Constitucional de Colombia, Sentencia T-517 de 1998.

Corte Constitucional de Colombia, Sentencia T-696 de 1996.

Corte Constitucional de Colombia, Sentencia T-787 de 2004.

Dow Chemicals vs United States, US Supreme Court (19 de mayo de 1986).

DOWNES, L. America can't lead the world in innovation if the FAA keeps dragging its feet on drone rules, The Washington Post, 8 de octubre de 2014. Recuperado de: http://www. washingtonpost.com/blogs/innovations/wp/2014/10/08/america-cant-lead-the-world-ininnovation-if-the-faa-keeps-dragging-its-feet-on-drone-rules/

Goldman v. United States, Us Supreme Court (27 de abril 27 de 1942).

Haw. Rev. Stat. Ann. § 711-1110.9 (2008).

Idaho Code Ann. § 18-6609 (2008).

JENKInS, D. y B. VASIGH. The Economic Impact of Unmanned Aircraft Systems Integration in the United States. Association of Unmanned Vehicle Systems International (AUVSI). Marzo de 2014. Recuperado de https://fortunedotcom.files.wordpress.com/2013/05/ new_economic_report_2013_full.pdf

JonEs, M. Drones the Sky's the Limit - Or is it? Technology and Engineering Teacher, 2014, p. 28.

Katz v. United States, US Supreme Court (18 de diciembre de 1967).

Kyllo v. United States, US Supreme Court (11 de junio de 2001). 
Ley Estatutaria 1621 de 2013.

Me. Rev. Stat. Ann. tit. 17-A, § 511 (2008).

O'BRIEN, J. Warrantless Government Drone Surveillance: A Challenge to the Fourth Amendment. Marshall J. Information Technology \& Privacy Law, vol. Xxx, 2013, p. 168.

Olmstead v. United States, US Supreme Court (4 de junio de 1928).

Otros autores (21 de febrero de 2009). El DAS sigue grabando. Semana. Recuperado de: http:// www.semana.com/nacion/articulo/el-das-sigue-grabando/100370-3

Quintero, J. Conozca los drones Made in Colombia. El Tiempo. 21 de abril de 2013. Recuperado de: http://www.eltiempo.com/archivo/documento/CMS-12753146

RAC 4.

SAlmon, S. A. Search and Seizure: Open field doctrine in lights of Katz - United States v. Basile. Arizona State Law Journal, 2-3, 1978, p. 366.

SCHLAG, C. New privacy battle: How the expanding use of drones continues to erode our concept of privacy and privacy rights. Pittsburgh Journal of Technology Law \& Policy. Vol. 13, 2013, p. 4.

Section 333 of the FAA Modernization and Reform Act of 2012 (FMRA).

Section 336 - Public Law 112-95.

Singer, P. W. Do drones undermine Democracy? The New York Times. 21 de enero de 2012. Recuperado de: http://www.nytimes.com/2012/01/22/opinion/sunday/do-drones-undermine-democracy.html?pagewanted=all\&_r=0

Smith v. Maryland, US Supreme Court (20 de junio de 1979).

The Bureau Investigative Journalism. Get the Data: Drone Wars: Casualty Estimates (actualizado a 10 de febrero de 2015). Recuperado de: http://www.thebureauinvestigates.com/ category/projects/drones/drones-graphs/

United States Code (49 U.S.C.).

VAlanis, K. P. Advances in Unmanned Aerial Vehicles: State of the Art and the Road to Autonomy. Dordrecht, Springer Science \& Business Media, 2007.

VILLASENOR, J. Observation from above: Unmanned Aircraft Systems and Privacy. Harvard Journal of Law \& Public Policy. Vol. 36, 2013, p. 36.

Wash. Rev. Code Ann. § 9A.44.115 (2008). 